\title{
Identification of a novel nonsense mutation in the rod domain of GFAP that is associated with Alexander disease
}

\author{
Tai-Seung Nam ${ }^{1,8}$, Jin Hee Kim ${ }^{2,3,8}$, Chi-Hsuan Chang ${ }^{4,8}$, Woong Yoon $^{5}$, Yoon Seok Jung ${ }^{6}$, Sa-Yoon Kang ${ }^{7}$, \\ Boo Ahn Shin ${ }^{3}$, Ming-Der Perng ${ }^{\star, 4,9}$, Seok-Yong Choi ${ }^{\star, 2,6,9}$ and Myeong-Kyu Kim ${ }^{\star 1,9}$
}

Alexander disease (AxD) is an astrogliopathy that primarily affects the white matter of the central nervous system (CNS). AxD is caused by mutations in a gene encoding GFAP (glial fibrillary acidic protein). The GFAP mutations in AxD have been reported to act in a gain-of-function manner partly because the identified mutations generate practically full-length GFAP. We found a novel nonsense mutation (c.1000 G $>$ T, p.(Glu312Ter); also termed p.(E312*)) within a rod domain of GFAP in a 67-year-old Korean man with a history of memory impairment and leukoencephalopathy. This mutation, GFAP p.(E312*), removes part of the 2B rod domain and the whole tail domain from the GFAP. We characterized GFAP p.(E312*) using western blotting, in vitro assembly and sedimentation assay, and transient transfection of human adrenal cortex carcinoma SW13 (Vim ${ }^{+}$) cells with plasmids encoding GFAP p.(E312*). The GFAP p.(E312*) protein, either alone or in combination with wild-type GFAP, elicited self-aggregation. In addition, the assembled GFAP p.(E312*) aggregated into paracrystal-like structures, and GFAP p.(E312*) elicited more GFAP aggregation than wild-type GFAP in the human adrenal cortex carcinoma SW13 $\left(\mathrm{Vim}^{+}\right)$cells. Our findings are the first report, to the best of our knowledge, on this novel nonsense mutation of GFAP that is associated with AxD and paracrystal formation.

European Journal of Human Genetics (2015) 23, 72-78; doi:10.1038/ejhg.2014.68; published online 23 April 2014

\section{INTRODUCTION}

Alexander disease (AxD; Online Mendelian Inheritance in Man (OMIM) \#203450), a neurodegenerative disease, is an astrogliopathy that primarily inflicts the white matter of the central nervous system (CNS). ${ }^{1-7}$ A characteristic pathological feature of $\mathrm{AxD}$ is the presence of Rosenthal fibers that are homogeneous eosinophilic inclusions stained with hematoxylin and eosin. Rosenthal fibers have been reported to possess GFAP (glial fibrillary acidic protein), $\alpha$ B-crystallin and heat shock protein 27 (HSP-27). However, the full composition of Rosenthal fibers remains undefined. ${ }^{2,6}$ Messing and colleagues ${ }^{8}$ reported in 2001 that $\mathrm{AxD}$ was caused by mutations in GFAP that encodes a type III intermediate filament (IF) predominantly found in astrocytes with the CNS. The GFAP consists of an N-terminal head, a central $\alpha$-helical rod and a C-terminal tail domain ${ }^{9}$ (Figure 1), and has several isoforms including $\alpha, \delta, \kappa, \Delta 135, \Delta 164$ and $\Delta$ exon6. ${ }^{10}$ Moreover, Messing and colleagues ${ }^{8}$ suggested that the GFAP mutations in $\mathrm{AxD}$ act in a gain-of-function manner because the phenotype of the Gfap null mouse was not similar to signs of $\mathrm{AxD}$ patients. Most of the GFAP mutations that have been identified in $\mathrm{AxD}$ are heterozygous, sporadic and missense mutations. ${ }^{6,11,12}$
Based upon statistical analysis of 215 cases of $\mathrm{AxD}$, Vanderver and colleagues $^{13}$ proposed in 2011 a new classification system for this disease. In the new system, type I AxD exhibits early age at onset (AAO), seizure, macrocephaly, encephalopathy, paroxysmal deterioration, failure to thrive, developmental delay and typical brain magnetic resonance imaging (MRI) features. Type II AxD, on the other hand, exhibits broad AAO, autonomic dysfunction, bulbar symptoms, ocular movement abnormality, palatal myoclonus and atypical MRI findings, and is frequently negative for neurocognitive or developmental deficits. ${ }^{13}$

Diagnostic workups of $\mathrm{AxD}$ include history taking, physical examination, brain MRI, sequencing of the GFAP and a cerebral biopsy. Of these workups, GFAP sequencing and cerebral biopsy are more conclusive. ${ }^{2,12}$

We saw a patient who presented with memory impairment, extensive cerebral white matter hyperintensities with a garland-like periventricular signal abnormalities on the brain $\mathrm{MR}$ images, and a nonsense mutation in a $2 \mathrm{~B}$ rod domain of the GFAP (c.1000 G $>\mathrm{T}$, p.(Glu312Ter); also termed p.(E312*)). To test whether the mutation is disease causing, we set out to determine whether GFAP p.(E312*) protein caused aggregation.

${ }^{1}$ Department of Neurology, Chonnam National University Medical School, Gwangju, Republic of Korea; ${ }^{2}$ Department of Biomedical Sciences, Chonnam National University Medical School, Gwangju, Republic of Korea; ${ }^{3}$ Department of Microbiology, Chonnam National University Medical School, Gwangju, Republic of Korea; ${ }^{4}$ Institute of Molecular Medicine, College of Life Sciences, National Tsing Hua University, Hsinchu, Taiwan; ${ }^{5}$ Department of Radiology, Chonnam National University Medical School, Gwangju, Republic of Korea; ${ }^{6}$ School of Biological Sciences and Technology, Chonnam National University, Gwangju, Republic of Korea; ${ }^{7}$ Department of Neurology, College of Medicine, Jeju National University, Jeju, Republic of Korea

*Correspondence: Professor M-D Perng, Institute of Molecular Medicine, College of Life Sciences, National Tsing Hua University, Hsinchu 30013 , Taiwan. Tel: +886 3 5742024; Fax: +886 3 5715934; E-mail: mdperng@life.nthu.edu.tw

or Professor S-Y Choi, Department of Biomedical Sciences, Chonnam National University Medical School, Gwangju 501-759, Republic of Korea. Tel: +82 62 220 4062, Fax: +82 62228 7294; E-mail: zebrafish@chonnam.ac.kr

or Professor M-K Kim, Department of Neurology, Chonnam National University Medical School, Gwangju 501-759, Republic of Korea. Tel:+82 62 220 6161 ; Fax:+82 62236 0839; E-mail:mkkim@chonnam.ac.kr

${ }^{8}$ These authors contributed equally to this work.

9 Joint senior authors.

Received 7 July 2013; revised 23 February 2014; accepted 5 March 2014; published online 23 April 2014 

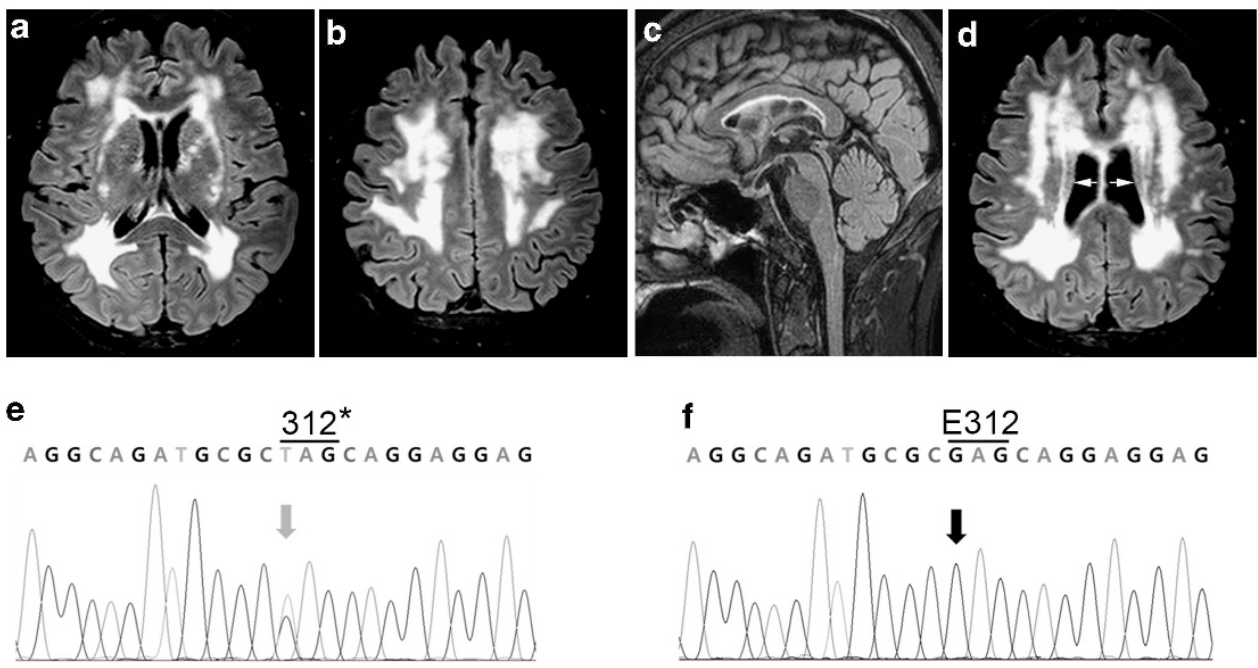

\section{f} A G G C A G A T G C G C $\overline{\text { G A G } C A ~ G ~ G ~ A ~ G ~ G ~ A ~ G ~}$
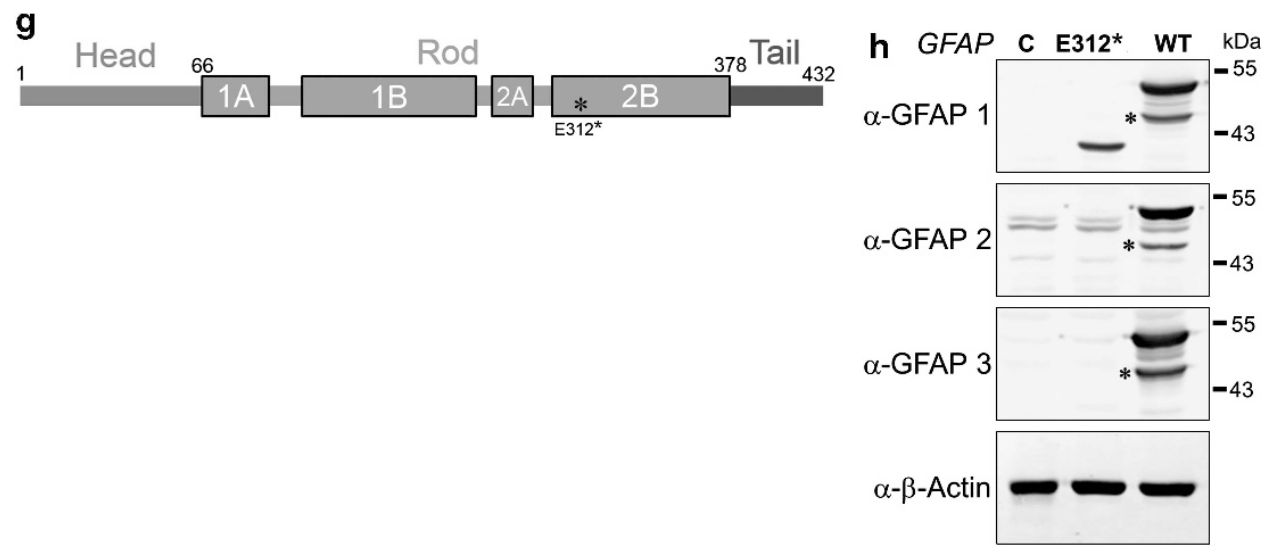

Figure 1 Radiological and genetic profiles of the proband. (a, b) Axial fluid-attenuated inversion recovery (FLAIR)-magnetic resonance images of the brain show extensive bilateral, hyperintense lesions in external capsules, periventricular (a) and deep cerebral white matters (b). (c) A sagittal FLAIR image shows no atrophic change or signal abnormalities in the medulla oblongata and upper cervical cord. (d) An axial FLAIR image shows garland-like signal intensities along the lining of the outer rim of the lateral ventricles (arrows). (e, f) DNA sequence analysis of the GFAP. Arrows indicate c.1000G. (e) Electropherogram of the proband reveals a previously unreported heterozygous G-to-T substitution at position 934 of the GFAP (c.1000G > T, p.E312Ter; Ter represents a Stop codon). (f) Representative electropherogram of the GFAP in 200 control subjects. (g) Schematic illustration of human GFAP. The numbers of the amino acid residues are according to $\mathrm{BC0} 13596$ (NCBI accession number). Asterisk indicates p.(E312*) mutation. Not drawn to scale. (h) Western blotting analysis of the GFAP p.(E312*) protein. HEK293T cells were transfected with plasmid encoding GFAP p.(E312*) and processed for WB with three different anti-GFAP antibodies. Antibody 1 was raised against the whole GFAP, and antibodies 2 and 3 were raised against the C-terminal half of GFAP. C, E312* and WT indicate untransfected, GFAP p.(E312*) and WT GFAP-transfected cells, respectively. $\beta$-Actin was used as a loading control. The asterisk represents degradation product of GFAP. Numbers to the right are molecular weight of protein standards in $\mathrm{kDa}$.

\section{MATERIALS AND METHODS}

\section{Subjects and ethics}

A 67-year-old Korean man with a history of leukoencephalopathy was referred to our hospital for an evaluation of his memory impairment. The patient had no medical history such as hypertension, ingestion of toxic substances or carbon monoxide poisoning except for a subtotal gastrectomy for early-stage gastric cancer at the age of 52 years. Brain MRI was performed at the age of 57 years to evaluate a sudden onset of severe headache and revealed extensive hyperintense signal changes in both the periventricular white matter regions and centrum semiovale on T2-weighted and fluid-attenuated inversion recovery (FLAIR) images (data not shown). Antiplatelet drugs were initiated under the impression of cerebral small vessels disease at that time. The patient had no family history of consanguineous marriage or neurological diseases including migraine, stroke and dementia.

At the age of 67 years, the proband's neurologic examination showed normal motor power and deep tendon reflexes in both arms and legs, and no abnormal brainstem signs were evident. He scored $26 / 30$ on a Mini-Mental Status Examination (MMSE), and a comprehensive neuropsychological test ${ }^{14}$ revealed deficits in his visual immediate and recent memory, naming and word fluency, along with mild depression. However, his daily living activities were normal.

The control group studied comprised 200 unrelated Korean individuals with no family history of AxD. Blood was drawn from this control group and the AxD patients from whom informed consents were obtained. The study protocol was approved by the Institutional Review Board at Chonnam National University Hospital, and was performed in accordance with the Helsinki Declaration of 1975.

\section{General reagents}

Pfu DNA polymerase and restriction endonucleases were purchased from Bioneer (Daejeon, Korea) and New England Biolabs (Ipswich, MA, USA), respectively. All other reagents were purchased from Sigma-Aldrich (St Louis, MO, USA) unless otherwise specified.

\section{DNA sequencing}

Genomic DNA was extracted from peripheral blood leukocytes of the subjects by phenol chloroform extraction. ${ }^{15}$ All nine exons and exon-intron boundaries 
of the GFAP were amplified from the genomic DNA by PCR using primers (Supplementary Table). The resultant amplicons were bidirectionally sequenced and then compared with the sequence of GFAP cDNA (GenBank accession number NM_002055.4). The DNA sequence of Notch3 was analyzed as described previously. ${ }^{16}$ The identified GFAP mutation, p.(E312*), was submitted to the 'Human Intermediate Filament Database' (http:// www.interfil.org).

Plasmid construction, cell culture, transfection, western blotting and preparation of cytoskeletal fractions

Detailed information is provided in the Supplementary Methods.

\section{Expression and purification of recombinant GFAP}

Both wild-type and mutant GFAP were expressed in Escherichia coli strain BL21(DE3) pLysS (EMD Millipore, Billerica, MA, USA) and purified as described previously. ${ }^{17}$ The expressed proteins were further purified by ion exchange chromatography using an AKTAprime plus system (GE Healthcare, Uppsala, Sweden) equipped with DEAE-Sepharose and CM-Sepharose Fast Flow columns. Peak fractions were analyzed by SDS-PAGE, and those containing the purified protein were collected and stored at $-80^{\circ} \mathrm{C}$. Protein concentrations were determined using BCA Protein Assay kit (Thermo Scientific, Rockford, IL, USA) with BSA as a standard.

\section{In vitro assembly and sedimentation assay}

In vitro assembly and sedimentation assays of GFAP were performed as described previously. ${ }^{17}$ In brief, purified GFAP was diluted to $0.3 \mathrm{mg} / \mathrm{ml}$ in $6 \mathrm{M}$ urea in buffer (10 mM Tris- $\mathrm{HCl}$ (pH 8.0), $5 \mathrm{~mm}$ EDTA, $1 \mathrm{~mm}$ EGTA and $1 \mathrm{~mm}$ DTT) and was dialyzed stepwise against $3 \mathrm{~m}$ urea in the buffer and then the buffer only. Subsequently, filament assembly was completed by dialyzing against assembly buffer (20 mM imidazole- $\mathrm{HCl}(\mathrm{pH} 6.8), 100 \mathrm{~mm} \mathrm{NaCl}$ and $1 \mathrm{~mm}$ DTT). The sedimentation assay was performed as described previously. ${ }^{18}$

\section{Electron microscopy}

GFAP in assembly buffer $(100 \mu \mathrm{g} / \mathrm{ml})$ was applied to glow-discharged carboncoated copper grids (Ted Pella Inc., Redding, CA, USA), negatively stained with $1 \%(\mathrm{w} / \mathrm{v})$ uranyl acetate (Electron Microscopy Sciences, Hatfield, PA, USA), and then examined by transmission electron microscopy (HT7700, Hitachi High-Technologies, Tokyo, Japan) with an accelerating voltage of $100 \mathrm{kV}$. Images were processed in Adobe Photoshop (Adobe Systems, San Jose, CA, USA). Filament length and diameter were measured on enlarged electron micrographs using ImageJ software (National Institutes of Health, Bethesda, MD, USA).

\section{Immunostaining of cells}

The GFAP in the human adrenal cortex carcinoma SW13 $\left(\mathrm{Vim}^{+}\right)$cells transfected with indicated plasmids was detected with immunocytochemistry using antiGFAP antibody (COVANCE, catalog no. SMI-21 R; Princeton, NJ, USA) and antivimentin antibody as described previously. ${ }^{19}$ The cells were then imaged with confocal laser scanning microscopy (LSM 510, Carl Zeiss, Jena, Germany).

\section{RESULTS}

\section{Clinical findings}

We sought to definitively diagnose the disease in the proband under a provisional diagnosis of acquired leukoencephalopathy. Among leukoencephalopathies, acquired CNS demyelinating diseases including multiple sclerosis, toxic leukoencephalopathy, progressive multifocal leukoencephalopathy and hypertensive leukoencephalopathy were excluded given the proband's stable clinical course, irreversible, nonprogressive MRI features and no history of hypertension, ingestion of toxic substances or carbon monoxide poisoning. Moreover, among hereditary leukoencephalopathies, both adrenoleukodystrophy and metachromatic leukodystrophy were also excluded because of negative serological tests for very long chain fatty acids and arylsulfatase A, respectively.
Another hereditary leukoencephalopathy, CADASIL (cerebral autosomal dominant arteriopathy with subcortical infarcts and leukoencephalopathy), was initially considered as a diagnosis because of the proband's history of chronic headache and the involvement of both external capsules on the brain MR image (Figure 1a). However, accumulation of Notch3 in the vessel wall, one of the typical diagnostic features of CADASIL, was not evident following immunostaining of skin biopsy with Notch3 monoclonal antibody. ${ }^{20,21}$ Furthermore, mutation analysis of all 33 coding exons and exonintron boundaries of Notch 3 was negative as well, thereby excluding CADASIL as a diagnosis.

The proband's brain MR images at the age of 67 years displayed extensive, bilateral hyperintensities in the periventricular and deep white matter (Figure 1a and b) that had no significant interval change compared with the MRI results taken at the age of 57 years. The atrophy and signal abnormalities in the medulla oblongata and upper cervical cord, the diagnostic features of adult onset $\mathrm{AxD},{ }^{22}$ were not noted (Figure 1c). Intriguingly, garland-like signal abnormalities along the lining of lateral ventricles were observed (Figure 1d, arrows). As a recent report proposed that scalloped, garland-like signal changes on the brain MR images 'constitute a new sign' of later onset $\mathrm{AxD},{ }^{23}$ we suspected $\mathrm{AxD}$ as a diagnosis, a leukoencephalopathy caused by GFAP mutation.

\section{Mutation analysis of the GFAP}

Analysis of the proband's GFAP revealed a heterozygous $G$ to $T$ substitution in exon 6 (a reference for exon numbering: GenBank RefSeqGene NG_008401.1) at position 934, causing an amino acid change at codon 312 (Glutamic acid to Stop, c.1000 G> T (reference cDNA sequence: GenBank NM_002055.4), p.(Glu312Ter) (p.(E312*)) (reference protein sequence: GenBank NP_002046.1)) (Figure 1e). This mutation was not found in any of our 200 normal Korean control subjects (Figure 1f), and PolyPhen-2 analysis (http://genetics. bwh.harvard.edu/pph2/) predicted that p.(E312*) would probably be damaging, thus suggesting pathogenic nature for this mutation. The proband's asymptomatic eldest daughter had the same GFAP p. $($ E312*) mutation, but other family members did not agree to undergo genetic analysis or brain MRI. As the proband's parents are deceased, we could not determine whether the p.(E312*) mutation was either de novo or hereditary.

\section{Expression of mutant GFAP in HEK293T cells}

E312 is located in the $2 \mathrm{~B}$ rod domain of the GFAP (Figure $1 \mathrm{~g}$ ). To test whether p.(E312*) mutation in the GFAP truncates and/or alters the stability of the protein, we first transfected plasmids encoding WT GFAP or GFAP p.(E312*) into HEK293 cells that express no endogenous GFAP. We then analyzed the GFAP protein by western blotting (WB) with three different antibodies against GFAP: antibody 1 was raised against the whole GFAP, whereas antibodies 2 and 3 were raised against the C-terminal half of the GFAP. WB with antibody 1 detected both WT GFAP and GFAP p.(E312*) with the respective expected molecular weight, yet the band intensity of GFAP p.(E312*) was lower than that of WT GFAP (Figure 1h, upper panel). As expected, antibodies 2 and 3 did not detect GFAP p.(E312*) as these antibodies recognize the C-terminal fragment of $\mathrm{GFAP}^{24}$ that is absent in GFAP p.(E312*) (Figure 1h, middle and lower panels). Taken together, these findings demonstrate that the p.(E312*) mutation truncates the GFAP and lowers expression levels of GFAP. 


\section{In vitro assay for aggregation of mutant GFAP}

To determine whether the GFAP p. $\left(\mathrm{E} 312^{*}\right)$ protein is prone to aggregation, recombinant WT and mutant GFAP were purified from E. coli, subjected to a dialysis-based in vitro assembly assay, negativestained with uranyl acetate and then imaged by electron microscopy. Whereas WT GFAP assembled into typical 10-nm filaments that were several $\mu \mathrm{m}$ in length (Figure $2 \mathrm{a})$, GFAP p.(E312*) failed to form extended filaments. Instead, the assembled proteins aggregated into paracrystal-like structure ${ }^{25}$ (Figure $2 \mathrm{~b}$, left and right panels) that exhibited an alternating light/dark banding pattern with an $\sim 22 \mathrm{~nm}$ axial repeat comprising an $\sim 2$-nm-wide darkly staining band and an $\sim 20 \mathrm{~nm}$-wide lightly staining band (Figure 2b'). This banding is reminiscent of paracrystals formed by lamins, ${ }^{26}$ Class $\mathrm{V}$ intermediate filaments. These paracrystal-like structures were often tapered at both ends (Figure 2b"), and occasionally had a clear fibril structure at a perturbed site in the paracrystal organization (Figure 2b"'). Moreover, as the proband had a heterozygous p.(E312*) mutation in GFAP, we next assessed the assembly behavior of 1:1 mixture of WT GFAP and GFAP p.(E312*). The 1:1 mixture formed filaments that were shorter and less uniform than those in the assembly of WT GFAP alone, and tended to aggregate markedly (Figure 2c, left and middle panels).
Moreover, as expression levels of GFAP p.(E312*) were lower than those of WT GFAP in cultured mammalian cells, we tested whether the 3:1 mixture of WT GFAP and GFAP p.(E312*) formed GFAP aggregation as well. Indeed, it aggregated GFAP markedly (Figure 2c, right panel).

To assess the extent to which filaments interact with one another in the whole filament population, we performed a low-speed centrifugation assay with WT GFAP or GFAP p.(E312*) alone or in combination. When assembled on its own, approximately one-seventh of the WT GFAP was found in the pellet fraction (Figure 2e, lanes 1 and 2). In contrast, almost all of the GFAP p.(E312*) was noted in the pellet fraction when assembled alone (Figure 2e, lanes 5 and 6). The percentage of sedimented GFAP in the 1:1 mixture of WT GFAP and GFAP p.(E312*) was between that found for each of these proteins alone (Figure 2e, lanes 3 and 4), indicating that WT GFAP and GFAP p. $\left(\right.$ E312 $\left.2^{\star}\right)$ copolymerize and that this copolymer does not aggregate to some extent. This is consistent with our previous report that GFAP filaments can incorporate a small portion of assembly-compromised GFAP. ${ }^{27}$ Finally, to determine the efficiency of the in vitro assembly of GFAP, we carried out a high-speed centrifugation assay with WT GFAP or GFAP p.(E312*) alone or in combination. The GFAP formed high-molecular-weight complexes under all of these conditions, as
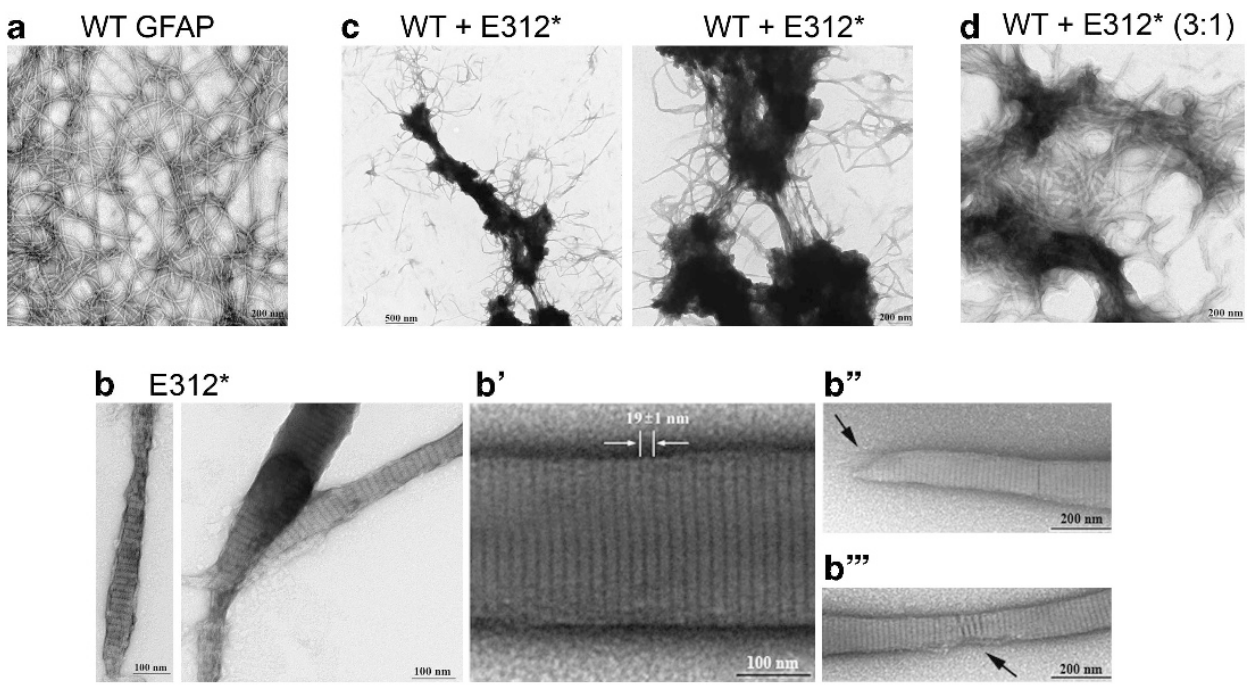

b'

b"
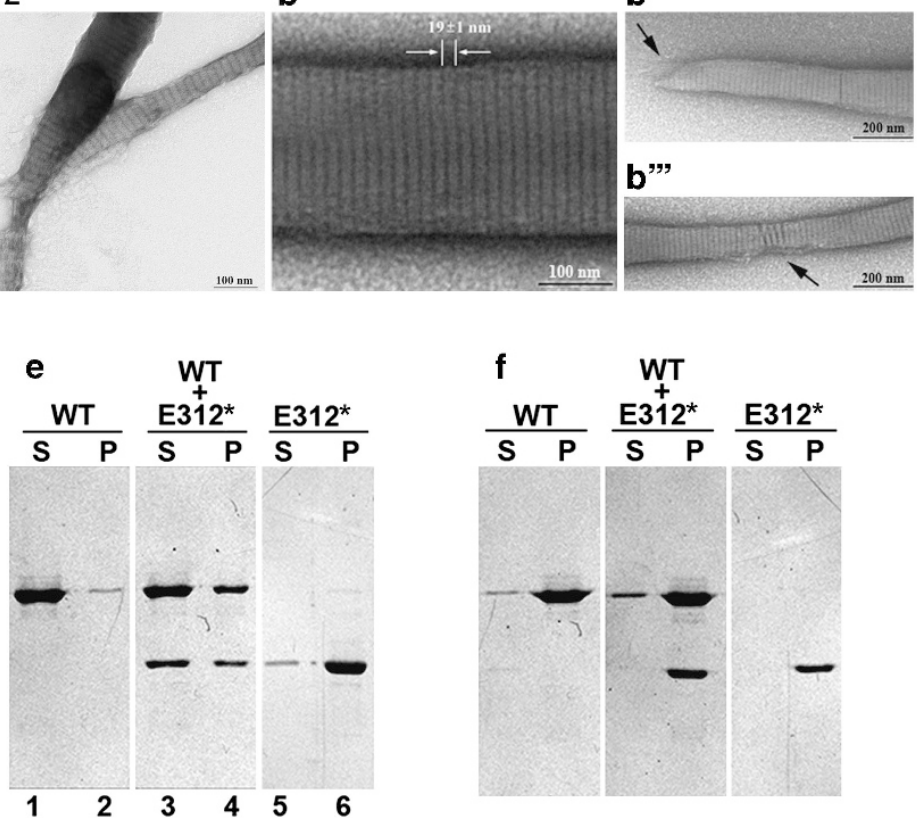

Figure 2 GFAP p.(E312*) is prone to aggregation. (a-d) Recombinant WT GFAP and GFAP p.(E312*) were purified from E. coli, assembled in vitro alone ((a) WT GFAP alone; (b) GFAP p.(E312*) alone) or in combination ((c) WT GFAP:GFAP p.(E312*) (1:1); (d) (3:1)), negative-stained with uranyl acetate and then imaged by electron microscopy. GFAP p.(E312*) formed paracrystal-like structures with distinctive $22 \mathrm{~nm}$ axial repeats with alternating light $(\sim 20 \mathrm{~nm})$ and dark ( $\sim 2 \mathrm{~nm}$ ) banding pattern (b'). The paracrystals were often tapered at both ends (b", arrow). The structural details of the aggregate were sometimes seen in less aggregated material, where a clear fibril structure was apparent at the perturbed site of the paracrystal organization (b'", arrow). (e, f) The assembled GFAPs were subjected to low-speed (e) and high-speed centrifugation (f), and the resulting supernatant (S) and pellet (P) fractions were visualized by SDS-PAGE and Coomassie Brilliant Blue staining. 
demonstrated by the presence of almost $90 \%$ of the total GFAP in the pellet fraction (Figure 2f). Collectively, these data suggest that the GFAP p.(E312*) promotes interfilament interactions that is dominant over the WT GFAP.

\section{GFAP p.(E312*) causes aggregation of GFAP in human adrenal cortex carcinoma SW13 $\left(\mathrm{Vim}^{+}\right)$cells}

To test whether GFAP p.(E312*) also caused GFAP aggregation in vivo, we transfected SW13 $\left(\mathrm{Vim}^{+}\right)$cells with plasmids encoding either WT GFAP or GFAP p.(E312*) or both. This cell line was derived from the human adrenal cortex carcinoma, features a vimentin filament network, expresses no endogenous GFAP and is well-suited for assessing mutant GFAP properties. ${ }^{17,24}$ Transfection efficiency of SW13 $\left(\mathrm{Vim}^{+}\right.$) cells was $\sim 26 \%$ in our hands (data not shown). Expressed WT GFAP assembled in $80 \%$ of the transfected cells into filamentous networks that partially coaligned with the vimentin IF networks (Figure $3 \mathrm{a}$ and $\mathrm{d}$ ) that is consistent with previous observations. ${ }^{17,24}$ In contrast, most of the cells $(73 \%)$ expressing GFAP p.(E312*) formed GFAP-rich aggregates that often collapsed the vimentin IF networks (Figure $3 \mathrm{c}$ and $\mathrm{d}$ ). Cotransfection with equimolar ratio of plasmids individually encoding WT GFAP and GFAP p.(E312*) resulted in GFAP aggregation with a frequency closer to that seen for cells transfected with GFAP p.(E312*) alone (Figure $3 \mathrm{~b}$ and $\mathrm{d}$ ). These results suggest that GFAP p.(E312*) not only impaired the ability of GFAP to form normal IF networks, but also exerted a dominant effect on the endogenous vimentin IF networks.

We demonstrated in GFAP-negative HEK293 cells that expression levels of GFAP p.(E312*) were lower than those of WT GFAP (Figure 1h). To assess expression levels and solubility of GFAP p. $\left(\right.$ E312 $\left.{ }^{\star}\right)$ further, we transfected SW13 $\left(\right.$ Vim $\left.^{+}\right)$cells with plasmids encoding WT GFAP or GFAP p.(E312*) or both, and the transfected cells were then lysed using a mild extraction protocol without deoxycholate and subjected to centrifugation. The total lysate and the resulting supernatant and pellet fractions were analyzed by WB with anti-GFAP antibody. Again, GFAP p.(E312*) amount was found to be lower than WT GFAP amount in the total lysates (lane 3, Figure $3 \mathrm{e})$, confirming that the $\mathrm{p} .\left(\mathrm{E} 312^{*}\right)$ mutation lowered expression levels of GFAP. Approximately $92 \%$ of the WT GFAP was detected in the pellet fraction whereas a small proportion $(\sim 8 \%)$ remained soluble. In contrast, GFAP p.(E312*) was found almost exclusively in the pellet fraction (lane 6 vs lane 12, Figure 3e), consistent with the formation of cytoplasmic aggregates. In the supernatant fractions, levels of WT protein in the co-transfected cells were lower than those in the WT GFAP alone transfected cells (lane $7 v s$ lane 6, Figure 3e), suggesting a dominant effect of GFAP p.(E312*) over WT GFAP.

\section{DISCUSSION}

Here, we show that a 67-year-old Korean man with extensive hyperintensities in the periventricular and deep white matter regions on MRI harbors nonsense p.(E312*) mutation in the GFAP, leading to deletion of part of the rod domain and whole tail domain in the GFAP. Moreover, we demonstrate that p.(E312*) mutation lowers expression levels of the mutant GFAP and promotes its aggregation.

GFAP mutations reported to date for $\mathrm{AxD}$ are (1) missense mutations in the coding region, (2) small insertions or deletions, (3) a splice-site mutation deleting exon 4 and (4) frameshift mutations at the extreme C-terminal end. ${ }^{3,13,28}$ Before the present study, a nonsense mutation in GFAP has not been reported for $\mathrm{AxD}$, to the best of our knowledge. The p.(E312*) mutation we reported here removes part of the $2 \mathrm{~B}$ rod domain and the whole tail domain from the GFAP. What is then the mechanism by which this truncated
GFAP induces the aggregation of GFAP? Herrmann and colleagues have shown that a tail domain of vimentin, another type III IF, is instrumental in the specification of the IF diameter. ${ }^{29}$ Moreover, Chen and Liem ${ }^{30}$ demonstrated that overexpression of GFAP lacking a whole tail domain in human adrenal cortex adenocarcinoma-derived SW13 cell lines elicits the formation of polymorphic aggregates, regardless of the presence or absence of vimentin, indicating an important role for the tail domain in the proper self-assembly of GFAP. Collectively, these reports suggest that as the tail domain is critical to the proper assembly of GFAP, ${ }^{10}$ the GFAP p.(E312*) mutant could not form proper GFAP networks, thereby leading to the formation of aggregates. However, the exact mechanism by which the tail domain facilitates proper assembly of GFAP warrants further study.

There have been robust MRI criteria for infantile and juvenile $\mathrm{AxD},{ }^{31}$ but MRI patterns for adult-onset $\mathrm{AxD}$ are variable, reflecting its clinical variability. ${ }^{23}$ Farina et al ${ }^{22}$ reported that atrophy in the medulla oblongata and upper cervical cord was present in MR images of $100 \%(11 / 11)$ of patients with genetically confirmed (all but one) adultonset $\mathrm{AxD}$. The MR images of the proband, however, did not display the atrophy in the medulla oblongata and upper cervical cord. Instead, the images exhibited extensive white matter hyperintensities. This discrepancy may ensue from the difference in the nature of GFAP mutations. Although GFAP mutations found in $\mathrm{AxD}$ to date do not significantly change the protein size of GFAP, the proband's GFAP has a nonsense mutation that deletes part of the $2 \mathrm{~B}$ rod domain and the $\mathrm{C}$-terminal tail domain. This discrepancy may account for the absence of characteristic brain stem symptoms of adult-onset $\mathrm{AxD}$ in the proband.

Although GFAP p.(E312*) was determined to be aggregation prone in vitro and in SW13 $\left(\mathrm{Vim}^{+}\right)$cells, we cannot completely exclude the possibility that the proband does not have AxD due to the lack of typical clinical signs and MRI findings. We postulate, however, that this is less likely owing to the following reasons. First, there have been reports on individuals who came to the clinic because of symptoms unrelated to $\mathrm{AxD}$ and were later found to harbor mutations in GFAP. $^{32-35}$ Second, even in these individuals, their MRI findings varied. For example, MRI of the patients 5 and 9 in the study of Farina et $a l^{22,34}$ revealed severe atrophy and signal changes in the brain stem and cervical spinal cord, whereas MRI of the patients no. 3 in Wada et $a l^{35}$ and in Okamoto et $a l^{33}$ displayed no such characteristic findings. Collectively, these reports suggest that $\mathrm{AxD}$ presents with diverse clinical symptoms and MRI findings. Furthermore, this report on GFAP p.(E312*) will expand the clinical repertoire of $\mathrm{AxD}$ even further.

Several coiled-coil fibrous proteins, such as myosin rod, ${ }^{36}$ paramyosin $^{37}$ and tropomyosin, ${ }^{38}$ tend to form paracrystals. In addition, Quinlan et $a^{25}$ demonstrated that mouse GFAP lacking the C-terminal tail domain, which still has the intact central $\alpha$-helical rod, forms paracrystals in vitro. Therefore, it was not surprising that of the GFAP mutations reported in AxD patients to date, GFAP p. $\left(\right.$ E312 $\left.2^{*}\right)$ lacking part of the $2 \mathrm{~B}$ rod domain and the C-terminal tail domain is the first mutant GFAP that formed paracrystals in vitro. Although the paracrystals described in the current study and that of Quinlan et al. ${ }^{25}$ were formed from the GFAP, there are several differences between them. For example, the length of an axial repeat in this report's paracrystals was much shorter than that in Quinlan et $a l^{25}: 22 \mathrm{~nm}$ vs $57 \mathrm{~nm}$. This difference may be the result of the difference in the size of GFAPs studied (GFAP lacking part of the 2B rod and the $\mathrm{C}$-terminal tail domains vs GFAP lacking the $\mathrm{N}$-terminal head and the C-terminal tail domains) and the difference in the condition under which GFAPs were assembled (standard assembly 

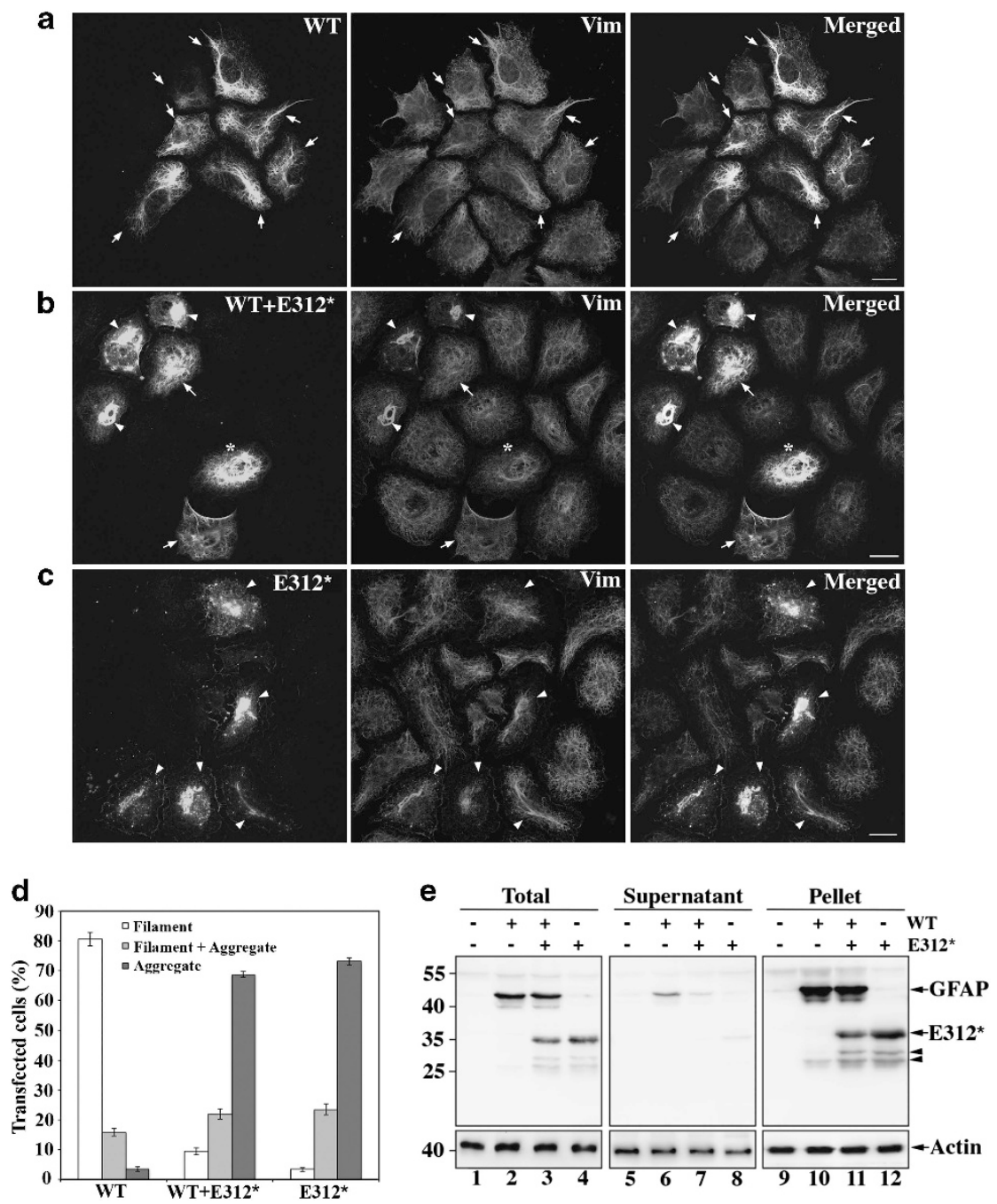

Figure 3 GFAP p.(E312*) causes aggregation of GFAP in adrenal cortex carcinoma SW13 $\left(\right.$ Vim $\left.^{+}\right)$cells. (a-c) SW13 (Vim $\left.{ }^{+}\right)$cells were transiently transfected with WT GFAP (a) or GFAP p.(E312*) (c), either individually or in combination in a 1:1 ratio (b). At $48 \mathrm{~h}$ after transfection, cells were immunostained with anti-GFAP (green channel) and anti-vimentin (red channel) antibodies, and imaged by confocal microscope. Merged images are shown in the right panels. Arrows indicate coalignment of GFAP and vimentin IF networks. Arrowheads represent GFAP aggregation colocalized with collapsed endogenous vimentin IF networks. Asterisk indicates coexistence of filament networks and aggregation. Scale bar $=10 \mu \mathrm{m}$. (d) The transfected cells were scored for their staining patterns (filament, filament + aggregate and aggregate) and shown as mean \pm SD. (e) SW13 $\left(V_{i m}^{+}\right.$) cells were transfected with plasmids encoding WT GFAP or GFAP p.(E312*) or both (1:1 plasmid ratio). The total amount of plasmid used was $8 \mu \mathrm{g}$ in all cases. Untransfected cells (lanes 1, 5 and 9) were used as a control. The cells were then lysed using a mild extraction protocol without deoxycholate and subjected to centrifugation. The resulting supernatant, pellet and total lysate were analyzed by WB with anti-GFAP antibodies. Anti-actin antibody was used as a loading control. Arrowheads indicate degradation fragments of GFAP. The full colour version of this figure is available at European Journal of Human Genetics online.

buffer at $\mathrm{pH} 7.0$ vs standard assembly buffer with divalent cations and chaotropic agents). Further study will be required to determine whether GFAP p.(E312*) forms paracrystals in vivo as well and whether this paracrystal formation is associated with the pathogenesis of AxD.

The finding that tailless GFAP precipitates aggregation furthers our understanding of the mechanism underlying aggregation of GFAP and the pathogenesis of $\mathrm{AxD}$.

\section{CONFLICT OF INTEREST}

The authors declare no conflict of interest.

\section{ACKNOWLEDGEMENTS}

We are grateful to the patient for his help and participation in the study. We thank Nan-Hee Choi for technical assistance, Seong-Min Choi for interpretation of comprehensive neuropsychological test, Hueng-Sik Choi for support, Gary Jenkins for English editing and Eun Young Choi for encouragement. This work was supported in part by grants from the Basic Science Research Program through the National Research Foundation of Korea (NRF) funded by the Ministry of Education (2013-R1A1A1058252), the Chonnam National University Hospital Biomedical Research Institute (CRI 12 055-21) and the National Science Council, Taiwan (99-2311-B-007-008-MY3). Tai-Seung Nam, Seok-Yong Choi and Myeong-Kyu Kim conceived the project.

\section{AUTHOR CONTRIBUTIONS}

Tai-Seung Nam, Jin Hee Kim, Chi-Hsuan Chang and Yoon Seok Jung performed the experiments. Tai-Seung Nam, Sa-Yoon Kang, Woong Yoon, Boo Ahn Shin, Ming-Der Perng, Seok-Yong Choi and Myeong-Kyu Kim analyzed and interpreted the data. Tai-Seung Nam, Ming-Der Perng, SeokYong Choi and Myeong-Kyu Kim drafted and revised the manuscript. 
1 Gorospe JR: Alexander Diesease [online]. Available at: http://www.ncbi.nlm.nih.gov/ books/NBK1172/. Accessed 21 Jan 2014.

2 Johnson AB, Brenner M: Alexander's disease: clinical, pathologic, and genetic features. J Child Neurol 2003; 18: 625-632.

3 Messing A, Brenner M, Feany MB, Nedergaard M, Goldman JE: Alexander disease. J Neurosci 2012; 32: 5017-5023.

4 Parpura V, Haydon PG: Astrocytes in (Patho)Physiology of the Nervous System. New York: Springer, 2009.

5 Quinlan RA, Brenner M, Goldman JE, Messing A: GFAP and its role in Alexander disease. Exp Cell Res 2007; 313: 2077-2087.

6 Sawaishi Y: Review of Alexander disease: beyond the classical concept of leukodystrophy. Brain Dev 2009; 31: 493-498.

7 Verkhratsky A, Sofroniew MV, Messing A et al: Neurological diseases as primary gliopathies: a reassessment of neurocentrism. ASN Neuro 2012; 4: e00082.

8 Brenner M, Johnson AB, Boespflug-Tanguy O, Rodriguez D, Goldman JE, Messing A: Mutations in GFAP, encoding glial fibrillary acidic protein, are associated with Alexander disease. Nat Genet 2001; 27: 117-120.

9 Reeves SA, Helman LJ, Allison A, Israel MA: Molecular cloning and primary structure of human glial fibrillary acidic protein. Proc Natl Acad Sci USA 1989; 86 5178-5182.

10 Middeldorp J, Hol EM: GFAP in health and disease. Prog Neurobiol 2011; 93: 421-443.

11 Messing A: Alexander disease [online]. Available at: http://www.waisman.wisc.edu/ alexander-disease/. Accessed 21 Jan 2014.

12 Mignot C, Boespflug-Tanguy O, Gelot A, Dautigny A, Pham-Dinh D, Rodriguez D: Alexander disease: putative mechanisms of an astrocytic encephalopathy. Cell Mol Life Sci 2004; 61: 369-385.

13 Prust M, Wang J, Morizono $\mathrm{H}$ et al: GFAP mutations, age at onset, and clinical subtypes in Alexander disease. Neurology 2011; 77: 1287-1294.

14 Ahn HJ, Chin J, Park A et al: Seoul Neuropsychological Screening Battery-dementia version (SNSB-D): a useful tool for assessing and monitoring cognitive impairments in dementia patients. J Korean Med Sci 2010; 25: 1071-1076.

15 Sambrook J, Russell DW: Molecular Cloning: A Laboratory Manual, 3rd edn. Cold Spring Harbor, NY: Cold Spring Harbor Laboratory Press, 2001.

16 Joutel A, Vahedi K, Corpechot C et al: Strong clustering and stereotyped nature of Notch3 mutations in CADASIL patients. Lancet 1997; 350: 1511-1515.

17 Der Perng M, Su M, Wen SF et al: The Alexander disease-causing glial fibrillary acidic protein mutant, R416W, accumulates into Rosenthal fibers by a pathway that involves filament aggregation and the association of alpha B-crystallin and HSP27. Am J Hum Genet 2006; 79: 197-213.

18 Nicholl ID, Quinlan RA: Chaperone activity of alpha-crystallins modulates intermediate filament assembly. EMBO J 1994; 13: 945-953.

19 Sandilands A, Prescott AR, Carter JM et al: Vimentin and CP49/filensin form distinct networks in the lens which are independently modulated during lens fibre cell differentiation. J Cell Sci 1995; 108:Pt 4 1397-1406.

20 Chabriat H, Joutel A, Dichgans M, Tournier-Lasserve E, Bousser MG: Cadasil. Lancet Neurol 2009; 8: 643-653.

21 Joutel A, Favrole $P$, Labauge $P$ et al: Skin biopsy immunostaining with a Notch3 monoclonal antibody for CADASIL diagnosis. Lancet 2001; 358: 2049-2051.

22 Farina L, Pareyson D, Minati $L$ et al: Can MR imaging diagnose adult-onset Alexander disease? AJNR Am J Neuroradiol 2008; 29: 1190-1196.

23 van der Knaap MS, Ramesh V, Schiffmann R et al: Alexander disease: ventricular garlands and abnormalities of the medulla and spinal cord. Neurology 2006; 66: 494-498.

24 Chen YS, Lim SC, Chen MH, Quinlan RA, Perng MD: Alexander disease causing mutations in the C-terminal domain of GFAP are deleterious both to assembly and network formation with the potential to both activate caspase 3 and decrease cell viability. Exp Cell Res 2011; 317: 2252-2266.

25 Quinlan RA, Moir RD, Stewart M: Expression in Escherichia coli of fragments of glial fibrillary acidic protein: characterization, assembly properties and paracrystal formation. J Cell Sci 1989; 93:Pt 1 71-83.

26 Aebi U, Cohn J, Buhle L, Gerace L: The nuclear lamina is a meshwork of intermediatetype filaments. Nature 1986; 323: 560-564.

27 Perng MD, Wen SF, Gibbon T et al: Glial fibrillary acidic protein filaments can tolerate the incorporation of assembly-compromised GFAP-delta, but with consequences for filament organization and alphaB-crystallin association. Mol Biol Cell 2008; 19. $4521-4533$.

28 Flint D, Li R, Webster LS et al: Splice site, frameshift, and chimeric GFAP mutations in Alexander disease. Hum Mutat 2012; 33: 1141-1148.

29 Herrmann H, Haner M, Brettel $M$ et al: Structure and assembly properties of the intermediate filament protein vimentin: the role of its head, rod and tail domains. J Mol Biol 1996; 264: 933-953.

30 Chen WJ, Liem RK: The endless story of the glial fibrillary acidic protein. J Cell Sci 1994; 107: Pt 8 2299-2311.

31 van der Knaap MS, Naidu S, Breiter SN et al: Alexander disease: diagnosis with MR imaging. AJNR Am J Neuroradiol 2001; 22: 541-552.

32 Balbi P, Seri M, Ceccherini I et al: Adult-onset Alexander disease: report on a family. J Neurol 2008; 255: 24-30.

33 Okamoto $\mathrm{Y}$, Mitsuyama $\mathrm{H}$, Jonosono $\mathrm{M}$ et al: Autosomal dominant palatal myoclonus and spinal cord atrophy. J Neurol Sci 2002; 195: 71-76.

34 Pareyson D, Fancellu R, Mariotti C et al: Adult-onset Alexander disease: a series of eleven unrelated cases with review of the literature. Brain 2008; 131: 2321-2331.

35 Wada Y, Yanagihara C, Nishimura Y, Namekawa M: Familial adult-onset Alexander disease with a novel mutation (D78N) in the glial fibrillary acidic protein gene with unusual bilateral basal ganglia involvement. J Neurol Sci 2013; 331: 161-164.

36 Safer D, Pepe FA: Axial packing in light meromyosin paracrystals. J Mol Biol 1980; 136: 343-358.

37 Cohen C, Szent-Gyorgyi AG, Kendrick-Jones J: Paramyosin and the filaments of molluscan 'catch' muscles. I. Paramyosin: structure and assembly. J Mol Biol 1971 56: 223-227.

38 Cohen C, Longley W: Tropomyosin paracrystals formed by divalent cations. Science 1966; 152: 794-796.

Supplementary Information accompanies this paper on European Journal of Human Genetics website (http://www.nature.com/ejhg) 